\title{
Extended ACTN Architecture to Enable End-To-End 5G Transport Service Assurance
}

\author{
Young Lee, Kaippallimalil John, Ricard Vilalta* \\ Huawei USA Research Center, Plano, TX, USA \\ * Centre Tecnològic de Telecomunicacions de Catalunya (CTTC/CERCA) \\ Tel: +1 (214) 244 7652, e-mail: leeyoung@huawei.com
}

\begin{abstract}
This paper is aimed to provide a novel approach for an end-to-end service assurance mechanism for 5G back-haul transport network. Abstraction and Control of Traffic Engineered (TE) Networks (ACTN) in an IETF standard architecture enabling virtual network operations tocontrol and manage large-scale multi-domain, multi-layer and multi-vendor TE networks, so as to facilitate network programmability, automation, efficient resource sharing. 3GPP 5G requirements calls for Network Slicing support for enhanced mobile broadband (eMBB) and for new 'use cases' that require massive machine-type communications (mMTC) and ultra-reliable and low latency communications (URLLC). In order to support these new requirements, the current 3GPP 5G architecture needs to support dynamic instantiation of end-to-end paths that assure traffic classes characterized by network slicing. This paper extends ACTN architecture as well as 3GPP 5G architecture to address service assurance and performance guarantee for an end-to-end $5 \mathrm{G}$ backhaul transport networks.
\end{abstract}

Keywords: 5G Transport, ACTN, CSO, Orchestration, Network Slicing, 3GPP, IETF

\section{INTRODUCTION}

Abstraction and Control of Traffic Engineered Networks (ACTN) framework defines the requirements, use cases, and an SDN-based architecture, relying on the concepts of network and service abstraction, detaching the network and service control from the underlying data plane. ACTN architecture encompasses Provisioning Network Controllers (PNCs), responsible for specific technology and administrative domains, orchestrated by MultiDomain Service Coordinator (MDSC) which, in turn, enables underlay transport resources to be abstracted and virtual network instances to be allocated to customers and applications, under the control of a Customer Network Controller (CNC) [1][2].

A network slice is defined by 3GPP as an end to end logical network comprising a set of managed resources and network functions [3]. Its definition and deployment starts from the RAN (Radio Access Network) and packet core, but in order to guarantee end to end SLAs (Service Level Agreements) and KPIs (Key Performance Indicators) especially for applications that require strict latency and bandwidth guarantee, the transport network also plays an important role and needs to be sliced as part of services bound to the different slices. However, it is not easy for clients/applications to interface directly with transport networks [4]. ACTN (Abstraction and Control of Traffic Engineered Networks) has been driving SDN standardization in IETF in the TEAS (Traffic Engineering and Signaling) WG with the emphasis of providing the desired customer interfaces that enable dynamic and automatic transport network slice instantiation and its life cycle operation [4]

\section{IETF ACTN VIRTUAL NETWORK SLICING SERVICE MODEL}

IETF ACTN VN model [5] discusses customer initiated virtual network slicing data model in which customer can control their virtual network slice to fit their needs. This model fulfills the key requirement: the ability for the customer to define and convey their virtual networks without having to understand transport network details [4]. This is for CNC - MDSC Interface of ACTN (CMI), as shown in Figure 1. This model describes VN YANG model for customer access points, virtual network access points, Virtual Network (VN) identifiers, its VNmembers, any constraints and policy customer cares for with respect to its VNs. Figure 1 shows the process of VN creation in the context of ACTN architecture. 


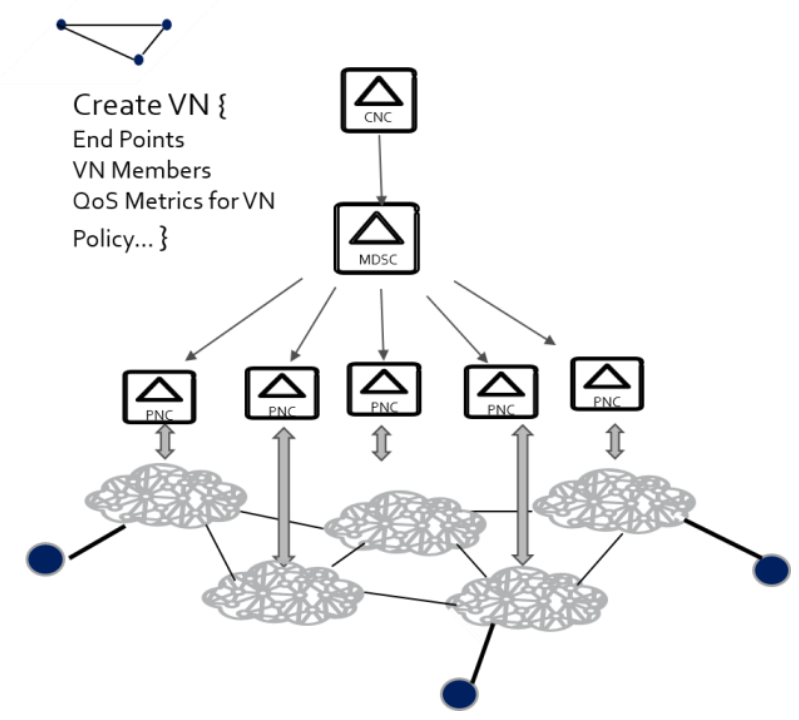

Figure 1. Virtual Network Slicing Service Creation

Figure 1 [2] shows that VN Slicing Service model enables customer to create its VN without having to know the transport underlay details and to indicate its end-points with constraints (e.g., bandwidth, latency, load-balancing, protection, etc.) per VN or VN-member level. This model facilitates customer-driven dynamic life-cycle VN service operation.

From a context of $5 \mathrm{G}$ transport network architecture, the CNC is the entity that is responsible for $3 \mathrm{GPP}$ access network coordination with the backhaul transport network. This entity is referred to as Traffic Provisioning Manager (TPM) for $3 \mathrm{GPP} / 5 \mathrm{G}$ context. The TPM is the creator of VNs and negotiates with the MDSC with regard to traffic class associated with each VN which in turn maps with network slice requirements. Thus, the TPM plays a central role from an orchestration point of view interacting with transport network's orchestration (i.e., MDSC) and with other TPMs in other domains. Section 3 provides details on the role of the TPM.

\section{3GPP 5G NETWORK ARCHITECTURE}

Mobile network backhauls in the past have used static configuration and provisioning of routers for traffic engineering (TE). These estimates maybe revised and TE is configured periodically based on demand and other performance criteria - however, this process takes a long time (in the order of weeks or months), thus may not be suitable for dynamically changing context such as $5 \mathrm{G}$ mobile network.

In 5G systems [6] [7] with a large range of services, low latency paths and mobility, the demand estimate varies much more dynamically (in the order of several minutes in the worst cases). Backhaul networks that provide capabilities to reprogram routers and switches to meet the new demand profile are needed. The base capability found in IETF ACTN [4] has been applied in the mobile network in [5].

In addition to the configuration and provisioning of traffic engineered paths between mobile and transport network providers, there is the question of how to enforce policies for slices, QoS across multiple transport network domains in mobile network and transport network. Each transport domain may employee different data plane technologies such as IP, MPLS, SR-MPLS, SRV6, OTN/WDM, etc. From an end-to-end 5G transport network perspective, it is paramount to ensure predictable and consistent service quality across all domains.

Figure 2 shows an enhanced 5G backhaul transport network architecture with a new function, which is referred to as Traffic Provisioning Manager (TPM). The TPM is deployed in each of the two domains/sites (Domain 1/Site 1, Domain 2/Site 2) and interfaces with other mobile network functions (e.g., Session Management Function (SMF), SDN Controller (SDN-C), etc.) while providing interfaces to transport network orchestrator (i.e., MDSC).

Figure 2 shows three segments/domains for $5 \mathrm{G}$ backhaul transport network.

- N3 segment/domain between Next Generation NodeB (gNB) and User Plane Function (UPF) is over the transport network at that site (Data Center/Central Office). 
- $\quad$ N9 mobile connection transport, there are three transport segments/domains - the transport at each mobile network site $(1,2)$ and the backhaul network in between.

- N6 transport segment between UPF and Application Servers (AS) is over the transport network at that site (Data Center/Central Office).

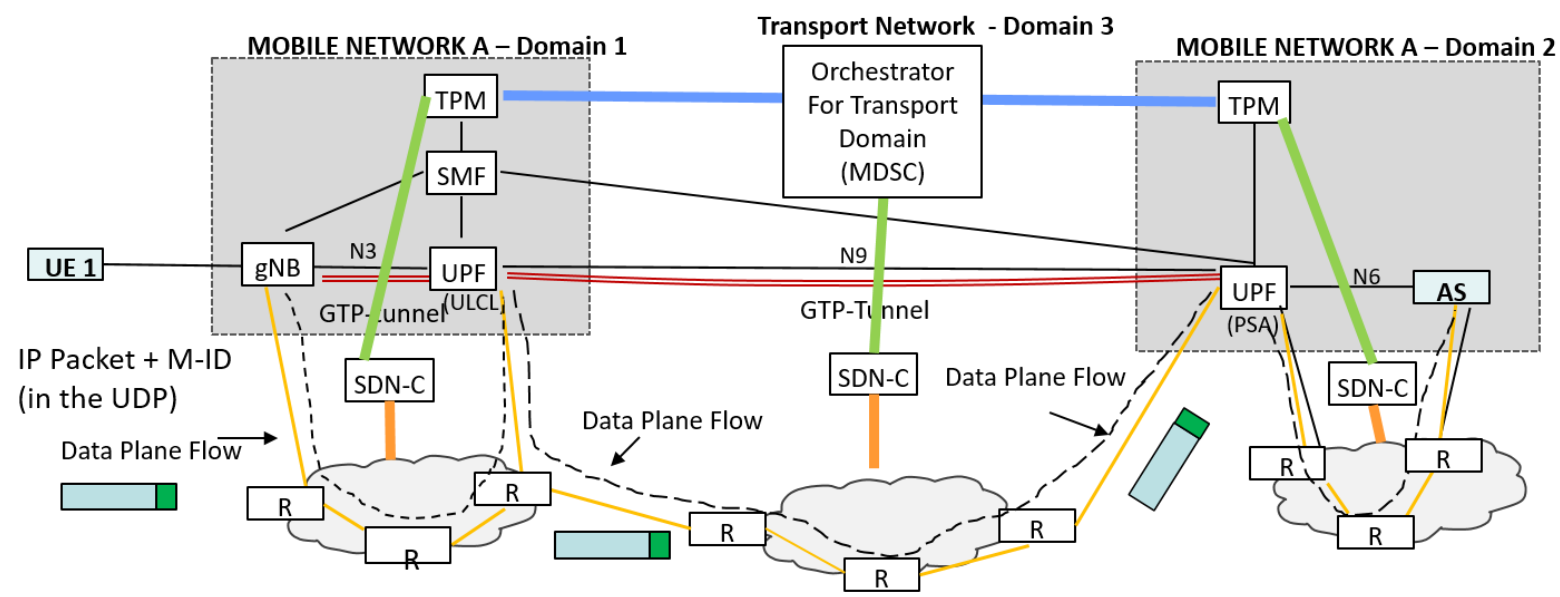

Figure 2. Enhanced 5G Backhaul Transport Network Architecture

The N3, N9 and N6 transport segments outlined in the figure are exemplary. For instance, in another case, the UPF PSA (PDN Session Anchor) and the AS may be in different sites. However, federated orchestration and controllers as described below is needed in general.

\section{FEDERATED ORCHESTRATION AND CONTROLLER FUNCTIONS}

The TPM is a type of the CNC as depicted in Figure 1. The TPMs and the MDSC form a federated orchestration relationship to each other in order to negotiate network slice policy and implement the negotiated network slice policy to its domain network, respectively. The TPM in Domain 1 is the initiator of the e2e network slice policy as it would estimate traffic matrix and determine service quality for each traffic class coupled with network slice requirement. This policy is referred to as Multi Transport Network Context (MTNC) and identified with MTNCID (M-ID). The MTNC-ID is allocated for each traffic class.

The MTNC identified by an MTNC-ID may include a set of requirements, such as quality of service (QoS) requirements, class of service $(\mathrm{CoS})$, a resilience requirement, and/or an isolation requirement, according to which transport resources of a transport network are provisioned for routing traffic between two service end points. The SDN controllers of each domain are responsible to create per class domain paths/VNs meeting the MTNC requirements. Once per class domain paths/VNs are created using ACTN VN model, the SDN controller would need to program the domain ingress router/network switch to populate the routing instruction so that the data packets associated with the MTNC-ID would be routed to the pre-established paths/VNs for the MTNC-ID.

\section{NETWORK PROGRAMMING FUNCTION OVER DATA PLANE}

There is a need to carry the MTNC-ID in data packets:

- Slices and QoS classes in the service domain do not have a 1:1 correspondence between the 3GPP domain and the transport domain. Some meta-data or token to associate information provisioned across $3 \mathrm{GPP}$ transport domains needs to be carried in the data packets that need to get specific treatment in the transport domain.

- The meta-data that is carried in the data packet header should be at the granularity of the provisioning for services between the 3GPP and transport domains. Specifically, the service is provided by the transport domain and the meta-data should be used in the transport domain to classify packets and provide the services agreed to. 
- Protocol extensions to carry the above policy meta-data across connection segments between 3GPP functions (N3, N9) and also across 3GPP - to external system (N6, e.g., to application server)

In order to support the data plane programming with MTNC-ID, the TPM would need to propagate MTNC-IDs to SMFs and, in turn, to gNBs/UPFs in its domain so that gNBs/UPFs would classify and "stamp" UDP flows with an MTNC-ID according to the QoS levels and slice information. Figure 2 shows that for N3, the data packets are "stamped" with the proper MTNC-ID by the gNBs via UDP header encapsulation mechanism. As for N9 and N6, the UPFs would need to stamp the data packets with the same MTNC-ID for the next domain. For each domain, all the packets identified by the MTNC-ID will be routed to the pre-established paths/VNs to ensure the proper level of service performance for the traffic class associated with the MTNC-ID.

Since the MTNC-IDs represent QoS and slice of the service domain that is mapped to a transport domain slice for a path between to network functions (NF), there are multiple flows that get mapped to a single such transport slice. The number of transport slices to be provisioned scales well as it is related to the number of sites $(\mathrm{N} *(\mathrm{~N}-1) / 2) * \mathrm{Q}$ for $\mathrm{N}$ number of sites, $\mathrm{Q}$ classes of service). For example, if there are 25 sites and 3 classes of service, the number of paths provisioned will at most be 900, while the number of PDN connection flows handled over those connections can be well over a million. As the number of transport paths setup is a few orders lower than the number of connections/flows that are handled, these mechanisms scale extremely well compared to setting this up per PDN connection.

\section{SUMMARY \& CONCLUSION}

This paper presented an extended ACTN architecture with 3GPP 5G transport architecture in order to provide a novel approach for an end-to-end service assurance mechanism to meet 3GPP 5G requirements for support of enhanced mobile broadband (eMBB) and for new 'use cases' that require massive machine-type communications (mMTC) and ultra-reliable and low latency communications (URLLC).

\section{REFERENCES}

[1] D. Ceccarelli and Y. Lee, "Framework for Abstraction and Control of Traffic Engineered Networks (ACTN)", RFC 8453, August 2018.

[2] R. Casellas, R. Vilalta, R. Martínez, R. Muñoz, H. Zheng, Y. Lee, "Experimental Validation of the ACTN architecture for flexi-grid optical networks using Active Stateful Hierarchical PCEs", ICTON 2017.

[3] 3rd Generation Partnership Project; Management and orchestration; Provisioning 3GPP TS 28.531

[4] D. Ceccarelli and Y. Lee, "Transport aspects of network slicing: existing solutions and gaps", IEEE Softwarization, January 2018.

[5] Y. Lee, et al, "A Yang Data Model for ACTN VN Operation”, draft-ietf-teas-actn-vn-yang-04, IETF draft, February 2019.

[6] 3rd Generation Partnership Project; Technical Specification Group Services and System Aspects; System Architecture for the 5G System; Stage 2 3GPP TS 23.501

[7] 3rd Generation Partnership Project; Technical Specification Group Services and System Aspects; Procedures for the 5G System; Stage 2 3GPP TS 23.502 